\title{
Contents
}

The Rest-Frame UV Spectrum of Elliptical Galaxies at High Redshift

Alvio Renzini

Rest-Frame UV and Visible Properties of Early Type Galaxies at $\mathbf{1}<z<\mathbf{2}$.

Patrick McCarthy.

Effect of Reionization on High-z Stellar Populations

Daniel Babich ............................... 19

Near UV Properties of Early-Type Galaxies at $z \sim 1$

Sperello di Serego Alighieri and Alessandro Bressan ............. 25

High Redshift Simulations Using the GALEX Ultraviolet Images of Nearby Galaxies

Bum-Suk Yeom, Young Kwang Kim, Soo-Chang Rey, Young Hoon Joe and Armando Gil de Paz......................... 31

GALEX Studies of Early-type Galaxies: the UV Rising Flux and Residual Star Formation

Michael Rich

UV Excess and AGB Evolution in Elliptical-Galaxy Stellar Populations

Rosa A. González-Lópezlira and Alberto Buzzoni ............... 51

Binary Stars as the Source of the Far-UV Excess in Elliptical Galaxies

Zhanwen Han, Philipp Podsiadlowski and Anthony E. Lynas-Gray...... 59 
Stellar Populations in Field Early-Type Galaxies

F. Annibali, A. Bressan, R. Rampazzo, P. Panuzzo W. Zeilinger

and L. Danese ..................................... 67

GALEX UV Observations of Elliptical Galaxies in Abell

Clusters at $z<0.2$

Chang H. Ree, Young-Wook Lee, Sukyoung Yi, Suk-Jin Yoon

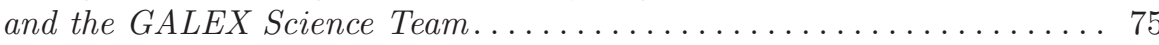

Near-UV Merger Signatures in Early-Type Galaxies

Jodie R. Martin, Robert W. O'Connell and John E. Hibbard........... 83

The Chemical Composition of Elliptical Galaxies Through

Mid-UV Indices

G. Ramos-Larios, M. Chávez, J. Morales-Hernández,

F. Huerta-Villapando, L. Sierra, H. de la Rosa, A. Pérez-Grana,

E. Bertone and L. H. Rodríguez-Merino ..................... 89

Colours of the Envelopes of cD Galaxies

S. N. Kemp, V. Guzmán Jiménez, P. Ramírez Beraud, J. A. Pérez

Grana, V. H. Ramírez Siordia, and F. J. Hernández Ibarra........... 93

A Morphological Study of M81: Comparison Between GALEX and Spitzer Imaging

I. Puerari, R. A. González-Lópezlira and M. Valdez-Gutiérrez........ 99

The Impact of Encounters on the Members of Local Group

Analogs. A View from $G A L E X$

Lucio M. Buson, Daniela Bettoni, Luciana Bianchi, Alberto Buzzoni, Antonietta Marino and Roberto Rampazzo.................... 105

GALEX UV Spectroscopy of Extended Objects: The Case of NGC 3079

Daniel Rosa González, Veronique Buat, Denis Burgarella,

Samuel Boissier, Alessandro Boselli and Armando Gil de Paz ........ 109

An Atlas of GALEX UV Spectra Selected from the CDFS Martha Bello-Ramírez and Daniel Rosa-González ................. 113

Weak X-ray Galaxies in the Chandra Deep Field South:

Searching for an Early Stage of Star Formation

Jesús López-Hernández, Daniel Rosa-González, Elena Terlevich

and Roberto Terlevich ................................ 117

UV Observations of Galactic Globular Clusters

Francesco R. Ferraro and Barbara Lanzoni ...................... 121 
Globular Clusters with the Extended Horizontal-Branch as Remaining Cores of Galaxy Building Blocks Young-Wook Lee, Hansung B. Gim and Chul Chung

The Blue Straggler Star Radial Distribution: Clues on Formation Mechanisms

Barbara Lanzoni

Infant Mortality of Star Clusters: on the Origin of the Diffuse UV Emission

Anne Pellerin, Martin J. Meyer, Jason Harris and Daniela Calzetti . . . 141

Are the Compact Star Clusters in M82 Evolving Towards Globular Clusters?

Y. D. Mayya, D. Rosa-González, L. H. Rodríguez-Merino, L. Carrasco,

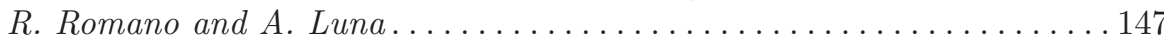

NGC 4214: Cluster Properties Derived from Multi-band Photometry

Leonardo Úbeda, Jesús Maíz-Apellániz, and John W. MacKenty........ 157

UV Spectroscopic Indices of Galactic Globular Clusters Javier Morales-Hernández, Miguel Chávez, Emanuele Bertone,

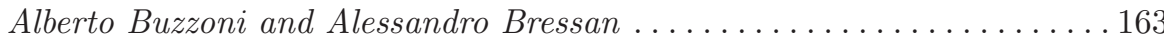

The Extended Emission of Ultracompact HII Regions: An Overview and New Observations Eduardo de la Fuente, Stanley E. Kurtz, M. S. N. Kumar, José Franco, Alicia Porras, Simon N. Kemp and Alfredo Franco-Balderas. . . . . . . . 167

Horizontal Branch Stars and the Ultraviolet Universe

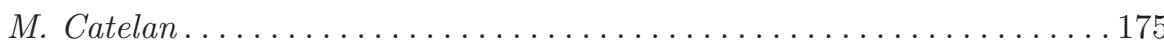

Subdwarf B and O Stars: Which Evolutionary Pathways?

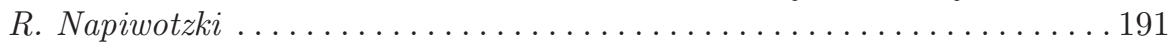

Computing Solar Absolute Fluxes

Carlos Allende Prieto

Recent Progress in Modeling Stellar Atmospheres

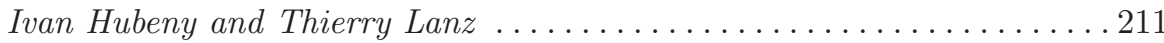

Ultraviolet Stellar and Cluster Spectra Compared to Theoretical Templates

Ruth C. Peterson

A Novel Precise Method for Correcting the Temperature in Stellar Atmosphere Models Octavio Cardona, Lucio Crivellari and Eduardo Simonneau 
New Model Atmospheres: Testing the Solar Spectrum in the UV

Lino H. Rodríguez-Merino, Octavio Cardona, Emanuele Bertone, Miguel Chávez and Alberto Buzzoni .......................2239

Photoionization, Recombination, and Radiative Transitions of Atoms and Ions

Sultana N. Nahar

Recombination rates, Resonance Strengths and Line Profiles of Dielectronic Satellite Lines of He-like Ca, Fe, Ni

Sultana N. Nahar, Justin Oelgoetz and Anil K. Pradhan ............ 259

Population Synthesis at Short Wavelengths and Spectrophotometric Diagnostic Tools for Galaxy Evolution Alberto Buzzoni, Emanuele Bertone, Miguel Chávez and Lino H. Rodríguez-Merino

Constraining Galaxy Evolution With Hubble's Next Generation Spectral Library

Sally Heap and Don J. Lindler

Spectral Evolution and Line Strength Indices in the UV

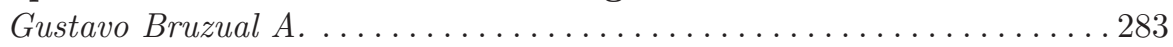

Synthesis Models in a Probabilistic Framework: Metrics of Fitting

Miguel Cerviño and Valentina Luridiana ...................... 293

World Space Observatory-UltraViolet: International Space Mission for the Nearest Future

Mikhail Sachkov, Ana I. Gómez de Castro, Isabella Pagano,

Francisco Torres, Yuri Zaiko and Boris Shustov ....................... 301

The Focal-plane Instruments on Board WSO-UV

Isabella Pagano, Mikhail Sachkov, Ana I. Gómez de Castro,

Maohai Huang, Norbert Kappelmann, Salvatore Scuderi,

Boris Shustov, Klaus Werner, and Gang Zhao ....................... 309

Science with the World Space Observatory - Ultraviolet Ana I. Gómez de Castro, Isabella Pagano, Mikhail Sachkov, Alain Lecavelier des Étangs, Giampaolo Piotto, Rosa González,

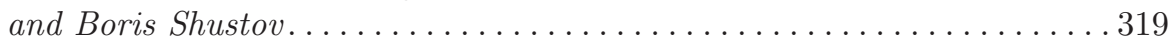

\section{Introducing UVIT}

Leonardo Úbeda, Carmelle Robert, Laurent Drissen, and the UVIT Canadian Science Team. . . . . . . . . . . . . . . . . . . . . . . . . . 329 
Efficiency of Selected UV Space Based Spectrometers

Valentina Klochkova, Vladimir Panchuk, Mikhail Sachkov,

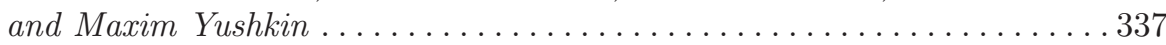

Astronomy at the University of Guadalajara

Miguel Chávez and Gerardo Ramos-Larios ..................... 341 